\title{
The Chaotic EM Algorithm based on the L-BFGS Operator and its Application in the Path Optimization
}

\author{
Liang Chen ${ }^{*}$
}

Institute of Information Science and Engineering, Qilu Normal University, Jinan 250200, P.R. China

\begin{abstract}
Aim at the class electromagnetic algorithm (EM) late for "mining" ability is insufficient, solution precision is not higher, and easy in premature problem, this paper proposes a combination of chaotic map and confined quasi-Newton (L-BFGS) local optimization operator of the chaotic class electromagnetism algorithm. Its main idea is in the late class electromagnetism algorithm using limit domain quasi-Newton operator to replace the class electromagnetism algorithm local optimization operator for local search; In the algorithm, the optimization process to join the chaos mapping, using chaos mapping of the random traversal characteristics, generate new individual and jump out of local to maintain the species diversity. The simulation through the simulation comparison of three consecutive field test functions, which show that the algorithm late can effectively jump out of local optimum, the basic type of electromagnetism algorithm has obvious advantages in convergence speed, a particle swarm optimization (PSO) algorithm and acceleration coefficient changing with time of particle swarm algorithm (TVAC) in terms of solution accuracy and fast convergence is better; Through the application in the path optimization results of comparison show that the algorithm is a cellular ant colony algorithm (ACO), particle swarm algorithm can get the best path in path optimization, it has better applicability in the discrete domain problem.
\end{abstract}

Keywords: Class electromagnetism algorithm, path optimization, the chaos map, test functions.

\section{INTRODUCTION}

The class electromagnetism algorithm (electromagnetism mechanism, EM) is a kind of between charged particles of attraction and repulsion setting up the mechanism in the electromagnetic field based on the coulomb law of the random search method [1]. The algorithm through the establishment of the individual by the size of the electric field force and the relationship between the fitness function value, according to the "excellent solution to attract poor solution, poor solution rejection excellent solution" as the principle of population movement trend and puts forward a kind of stochastic global optimization heuristic algorithm [2]. In the country has been successfully applied to distribution network fault location [3], assembly line assembly [4] and other aspects. But the basic EM algorithm the same with other global intelligent algorithm, has premature convergence and later local search accuracy is not higher, the defects of slow convergence speed [1]. Aimed at the shortcoming of the EM algorithm, this paper puts forward algorithm to improve the local optimization strategy, adopts the high precision local optimization operatorconfined quasi-Newton operator [5] (Limited-memory BROYDEN-FLETCHER-GOLDFARB-SHANNO, L-BFGS), taking to the late algorithm to the optimal individual near solution domain for optimal; and uses the chaos mapping [6], increase the diversity of species. Particle Swarm algorithm [7] (Particle Swarm Optimization, PSO) as a more mature intelligent algorithm, in the optimization of continuous domain has very good effect, its improved algorithm, such as acceleration coefficient changing with time of Particle Swarm algorithm (Time-varying accelerator coefficients Particle Swarm Optimization, TVAC) [7-9] better optimization ability, thus used as a comparison with the algorithm of this paper, the simulation results show that LBFGS-EM algorithm is the basic type of electromagnetic (EM) algorithm in convergence speed and better ability to jump out of local optimal performance, comparison with the Particle Swarm Optimization (PSO) algorithm and the acceleration coefficient changing with time of Particle Swarm Optimization (PSO) algorithm in terms of solution accuracy and fast convergence is better; By in the path planning problem (0-1) application results show that the LBFGS-EM algorithm can search the best path, comparison with the genetic algorithm, particle swarm algorithm has better adaptability in discrete domain problem.

\section{CLASS ELECTROMAGNETISM ALGORITHM}

The basic EM algorithm is composes of initialization, local search and resultant force calculation, the particle movement and termination of judgment of five parts.

1) Initialization: Initialization process of the EM algorithm is a random initialization process, namely in the solution domain randomly generated several solution as the original. The particle $\boldsymbol{X}_{\boldsymbol{i}, \boldsymbol{j}}$ charge value of the expression is: 


$$
\boldsymbol{q}_{k, i}=\exp \left(-n \frac{f\left(\boldsymbol{X}_{k, i}\right)-f\left(\boldsymbol{X}_{k, b e s t}\right)}{\sum_{i=1}^{m}\left(f\left(\boldsymbol{X}_{k, i}\right)-f\left(\boldsymbol{X}_{k, b e s t}\right)\right)}\right)
$$

In the formula: $\boldsymbol{q}_{k, i}$ shows in the $k$ iteration process of the $i$ particles with the charge value; $n$ shows the total number of particles, $m$ shows total number of particles in a population; $f(\bullet)$ shows evaluation function; $\boldsymbol{X}_{\boldsymbol{k}, \boldsymbol{i}}$ shows in the $k$ iteration process of the $i$ particles; $\boldsymbol{X}_{k \text {, best }}$ shows in the $k$ iteration process of the best particle in the evaluation function value.

2) Local search: EM algorithm local search strategy using linear search, and its expression as shown [2] in formula (2):

$\boldsymbol{Y}_{i}=\left\{\begin{array}{l}\boldsymbol{X}_{i}+\alpha\left(U-\boldsymbol{X}_{i}\right), \alpha>0.5 \\ \boldsymbol{X}_{i}-\alpha\left(\boldsymbol{X}_{i}-L\right), \alpha \leq 0.5\end{array}\right.$

In the formula (2), $\boldsymbol{X}_{i}$ shows the $i$ particles; $Y_{i}$ shows the particles $\boldsymbol{X}_{i}$ search within the territory of particles; $\alpha$ shows $0-1$ of the random number; $L$ shows feasible region of the lower boundary; $U$ shows feasible region of the upper boundary.

3) Resultant force calculation: According to the principle of superposition, the calculating formula of each particle $\boldsymbol{X}_{i}$ by the force $F_{i}$ can be defined as shown [1] in formula (3).

$\boldsymbol{F}_{i}=\sum_{j \neq i}^{m}\left\{\begin{array}{l}\frac{q_{i} q_{j}\left(\boldsymbol{X}_{j}-\boldsymbol{X}_{i}\right)}{\left\|\boldsymbol{X}_{j}-\boldsymbol{X}_{i}\right\|^{2}}, \quad f\left(\boldsymbol{X}_{j}\right)<f\left(\boldsymbol{X}_{i}\right) \\ -\frac{q_{i} q_{j}\left(\boldsymbol{X}_{j}-\boldsymbol{X}_{i}\right)}{\left\|\boldsymbol{X}_{j}-\boldsymbol{X}_{i}\right\|^{2}}, f\left(\boldsymbol{X}_{j}\right) \geq f\left(\boldsymbol{X}_{i}\right)\end{array}\right.$

Can be seen from the resultant force calculation formula, the better function value of particle will attract the poorer function value of particle, the objective function value of less particles will reject better function value of particle, if $f\left(\boldsymbol{X}_{j}\right)<f\left(\boldsymbol{X}_{i}\right)$, then it shows the appeal between the others, on the contrary, it shows the repulsive force, and the direction of the force between any two particles always points to the better objective function value of particles, so as to ensure the algorithm able to find the optimal solution finally.

4) The particles movement: The formula of particle $\boldsymbol{X}_{i}$ mobile can be defined as:

$\boldsymbol{Z}_{i}=\boldsymbol{X}_{i}+\lambda \frac{\boldsymbol{F}_{i}}{\left\|\boldsymbol{F}_{i}\right\|}$

Among them, $\lambda$ shows random step length, and scope for decimal number between $0 \sim 1, \boldsymbol{Z}_{i}$ shows the new particles produced after moving, after moving, recalculate the size of $f\left(\boldsymbol{Z}_{i}\right)$ and $f\left(\boldsymbol{X}_{i}\right)$, and take the greedy selection.
5) Termination of judgment: As with the same of the rest intelligent algorithm, set the iteration terminating condition, meet the termination, continue to iteration conversely.

Sketch design system as a CAD system in the expression and interaction [6,7], has a lot of inconvenience because of the interactive tools and technical limitations. Most CAD systems require users to provide more accurate information and steps to interact more cumbersomely, thus affecting the natural expression of design intention. In addition, it could not sustain the concept design very well and the seamless chaining between achieved and detailed design. Nowadays, sketch design is more and more accepted and used, because of the property it has it was an idealistic communication mode, and it could communicate in the different level of abstraction at the same time. There are more means and skills to support the human-computer communication, we can draw the sketch on the basis of different use or purpose, the introduction of the development of pen-based user interface and new interactive technology has provided an efficient and natural way of sketch design.

\section{THE FUSION L-BFGS OF THE CHAOTIC CLASS ELECTROMAGNETISM ALGORITHM}

\subsection{The Confined Quasi-Newton Local Operator}

Consider formula $(3,4)$ calculation method of the resultant force, if the individual $i_{\text {close to the optimal }}$ individual $\boldsymbol{X}_{\text {best }},\left|\boldsymbol{x}_{i}-\boldsymbol{x}_{j}\right| \rightarrow 0$, then $\left|\boldsymbol{F}_{i}\right| \rightarrow \infty$, making the individual $i$ resultant force is very big, the direction of the particle movement can produce larger error, near optimal solution cannot be searched. So when encounter this situation, this article adopts the confined quasi-Newton method (Limited memory BROYDEN-FLETCHERGOLDFARB-SHANNO, L-BFGS) for local search, the kind of the method is effective to use derivative of the best optimization operator, has the convergence speed fast, the advantages of strong local search ability. Setting the $\boldsymbol{S}_{k}=\boldsymbol{X} k+1-\boldsymbol{X} k, \boldsymbol{y}_{k}=\nabla f\left(\boldsymbol{x}_{k+1}\right)-\nabla f\left(\boldsymbol{x}_{k}\right)$, then Hessen matrix is deduced as follows (5). The specific derivation process reference the literature [10].

$$
\begin{aligned}
& \boldsymbol{H}_{k+1}=\boldsymbol{V}_{k}^{\mathrm{T}} \boldsymbol{H}_{k} \boldsymbol{V}_{k}+\rho_{k} \boldsymbol{S} \boldsymbol{s} \boldsymbol{S}_{k}^{\mathrm{T}} \\
& =\boldsymbol{V}_{k}^{\mathrm{T}}\left[\boldsymbol{V}_{k-1}^{\mathrm{T}} \boldsymbol{H}_{k-1} \boldsymbol{V}_{k-1}+\rho_{k-1} \boldsymbol{S}_{k-1} \boldsymbol{S}_{k-1}^{\mathrm{T}}\right] \boldsymbol{V}_{k}+\rho_{k} \boldsymbol{k} k \boldsymbol{S}_{k}^{\mathrm{T}} \\
& =\ldots \\
& =\left[\boldsymbol{V}_{k}^{\mathrm{T}} \ldots \boldsymbol{V}_{k}-\hat{m}+1\right] \boldsymbol{H}_{k-\hat{m}+1}\left[\boldsymbol{V}_{k}-\hat{m}+1 \ldots \boldsymbol{V}_{k}\right]
\end{aligned}
$$

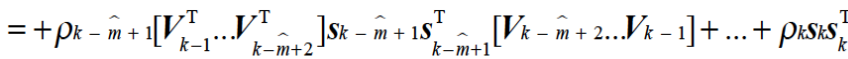

So the quasi-Newton operator steps as follows:

1) Selecting the initial node $x_{o} \in R^{n}$ and setting up positive integer $\mathrm{m}$;

2) Setting $H_{0}=I, k=0$, calculate the objective function $\mathrm{f}(\mathrm{x})$ in $x_{k}$ of the gradient; 
3) Determining the search direction $d_{k}$, make $\boldsymbol{d}_{k}=\mathrm{H}_{k} \boldsymbol{g}_{k}$

4) Starting from $x_{k}$, along the direction of $d_{k}$ searches, to solve $\lambda_{k}: x_{\text {best }}=\left(x_{1}, x_{2}, \ldots x_{n}\right)$;

5) Making $x_{k+1}=x_{k}+\lambda_{k} d_{k}$; if $\quad x_{\text {best }}=\left(x_{1}, x_{2}, \ldots x_{n}\right)$, then stop the iteration, get the optimal solution, otherwise continue to step 6 ;

6) Taking $x_{\text {best }}=\left(x_{1}, x_{2}, \ldots x_{n}\right)$, according to the formula (5) update $m$ times $\mathrm{H} 0$ and get $H_{k}$.

\subsection{The Chaos Operator}

Considering the chaotic movement has a good diversity, so using the chaotic operator for local search can be improve the performance of the algorithm [11]. Specific operation is as follows:

Making Up and Low respectively feasible region upper and lower bounds, $\boldsymbol{x}_{\text {best }}=\left(x_{1}, x_{2}, \ldots x_{n}\right)$ shows the state of the current optimal position. Then, its maps process first as shown in the formula (6) of the mapping rules:

$\alpha=\frac{\boldsymbol{x}_{\text {best }}-L o w}{U p-L o w}$

Obviously, after mapping interval is $[0,1]$, so this paper can be generated chaotic variables $[12,13]$ by using Logistic mapping as shown in formula (7):

$\alpha^{\prime}=\mu \alpha(1-\alpha)$

$x_{b e s t}^{\prime}=L o w+\alpha^{\prime}(U p-L o w)$ (8) In the formula, $\mu$ shows control parameters, if value is 4 , the system is in a state of complete chaos, is conducive to jump out of local optimum.

Finally, as shown in formula (8), take the chaotic variable inverse mapping to the desires of solution domain, so process over and over again, until the function value no longer changes.

\subsection{Building LBFGS - EM Algorithm}

Algorithm specific steps as follows:

Step 1: Initialization.

Step 2: Local search. According to the formula (2) take the local search and greedy choice around the initial solution.
Step 3: Determine whether the confined quasi-Newton method and local optimization. Namely the setting threshold $\varepsilon$, when $\left|x_{i}-x_{j}\right|<\varepsilon$, then the following random draw on $\mathrm{k}$ times the confined quasi-Newton operator to be obtained with a local optimization, and jump to the steps:

Step 4: Otherwise enter the next step.

Step 5: Resultant force calculation. According to the formula (3) and the formula (4), the particles of the resultant force are calculated separately, particles move fitness function value.

Step 5: The particle movement. Calculating the step4 individual fitness value, and compared with the previous generation optimal individual, if $f\left(\boldsymbol{X}_{i}^{\prime}\right)>f\left(\boldsymbol{X}_{i}\right)$, then take the greedy choice; otherwise enter the chaotic mapping process:

1) Setting the chaotic search iterative threshold IBET;

2) Using the formula (6) shown in the rules, will existing particle mapped to the chaotic space;

3) For variable after the mapping, adopt rules shown in formula (7) to create new chaotic variables;

4) Will produce chaotic variables using the formula (8) reflect the shooting method, projection solution domain, get new particle;

5) If it gets new particles fitness is greater than the original particles, then takes the greedy choice, otherwise remain the same.

Step 6: Termination of the judgment. Determine whether the number of iterations to satisfy the initial conditions set the threshold. If it is not satisfy, then return to Step 2; if it is meet, then the end of the iteration, output results.

\section{THE SIMULATION INSTANCE}

\subsection{Test Function Simulation}

This paper use test function of three consecutive domains [14]: SPHERE(f1) ,GRIEWANK(f2) ,ROSENBROCK(f3) under the MATLAB2010a environment, $2.13 \mathrm{GHz}$ processor, memory is $4 \mathrm{~GB}$ of $\mathrm{PC}$ for algorithm simulation.

In order to compare the improved algorithm to the superiority of the algorithm, this paper takes the basic EM algorithm and the proposed algorithm for the above three continuous domain test function simulation test 100 times, including function $\mathrm{f} 1$ iterations times take 1000 generation, function $\mathrm{f} 2$ due to need to judge the ability of jump out of

Table 1. Convergence iterations times contrast table.

\begin{tabular}{|c|c|c|c|}
\hline Algorithm & $\boldsymbol{f}_{\mathbf{1}}$ Convergence Iterations Times & $\boldsymbol{f}_{\mathbf{2}}$ Convergence Iterations Times & $\boldsymbol{f}_{3}$ Convergence Iterations Times \\
\hline \hline LBFGS-EM algorithm & 854 & 987 & 1520 \\
\hline EM algorithm & 901 & 1685 & 4000 \\
\hline PSO algorithm & 2380 & 3578 & 4000 \\
\hline TVAC algorithm & 1709 & 2978 & 4000 \\
\hline
\end{tabular}


local optimal, so the number of iterations take longer, for 6000 generations, $\mathrm{f} 3$ for 4000 generations; dimension take 30 , the number of populations take 50 . Taking one of the most optimal solutions for statistical comparison, get the results shown in Table 1, the EM data taken from the literature [1]. Apply the improved algorithm of optimal results compared with before improved, get the iterative contrast diagram as shown in (Figs. 1-3).

Can be seen from (Fig. 1), the EM algorithm and LBFGS-EM algorithm in Sphere test function optimization can effectively in the search for optimal solution, but the latter has been met to quasi-Newton local optimization operator after setting threshold, which can be more rapid, accurate for local optimization, at the end of the iteration solution precision is higher than the EM algorithm.
Can be found from (Fig. 2) contrast, for GRIEWANK function optimization process, the performance of the LBFGS-EM algorithm is higher than the EM algorithm, analysis the reason that the GRIEWANK function is a multimodal function, more the number of local optimum, so introduce quasi-Newton operator better searching local optimal solution, and then through the chaos mapping jump out the local optimum, which reflects the good performance.

Can be found from (Fig. 3), the EM algorithm in the ROSENBROCK function optimization on performance is not ideal, because it is difficult to discern the search direction, and after introducing chaos strategy, once appear the optimal solution, then stop updating phenomenon, through the chaotic mapping to generate new solutions, which is leading to some extent to search in the direction of

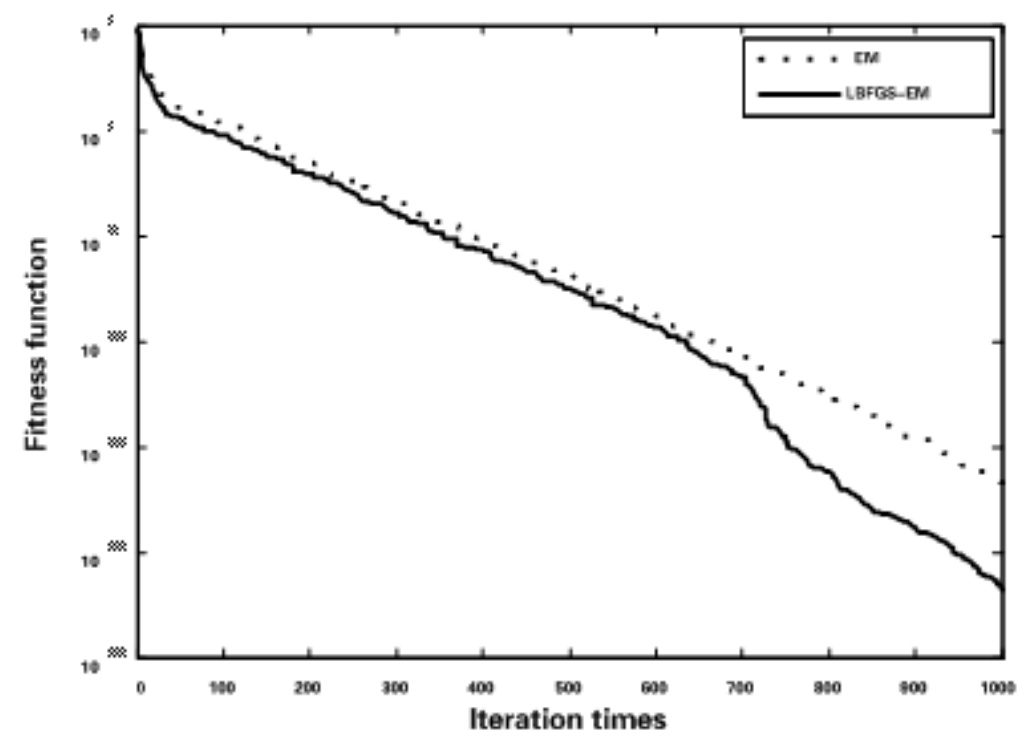

Fig. (1). SPHERE test function iteration convergence curve contrast figure.

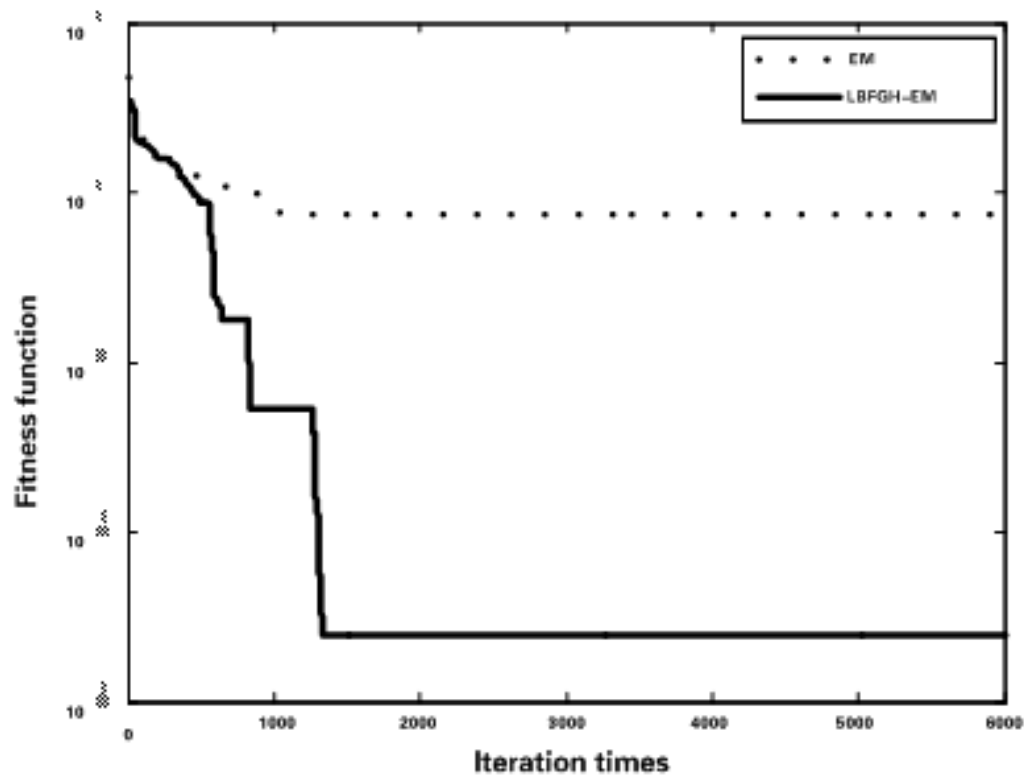

Fig. (2). GRIEWANK test function convergence curve contrast figure. 


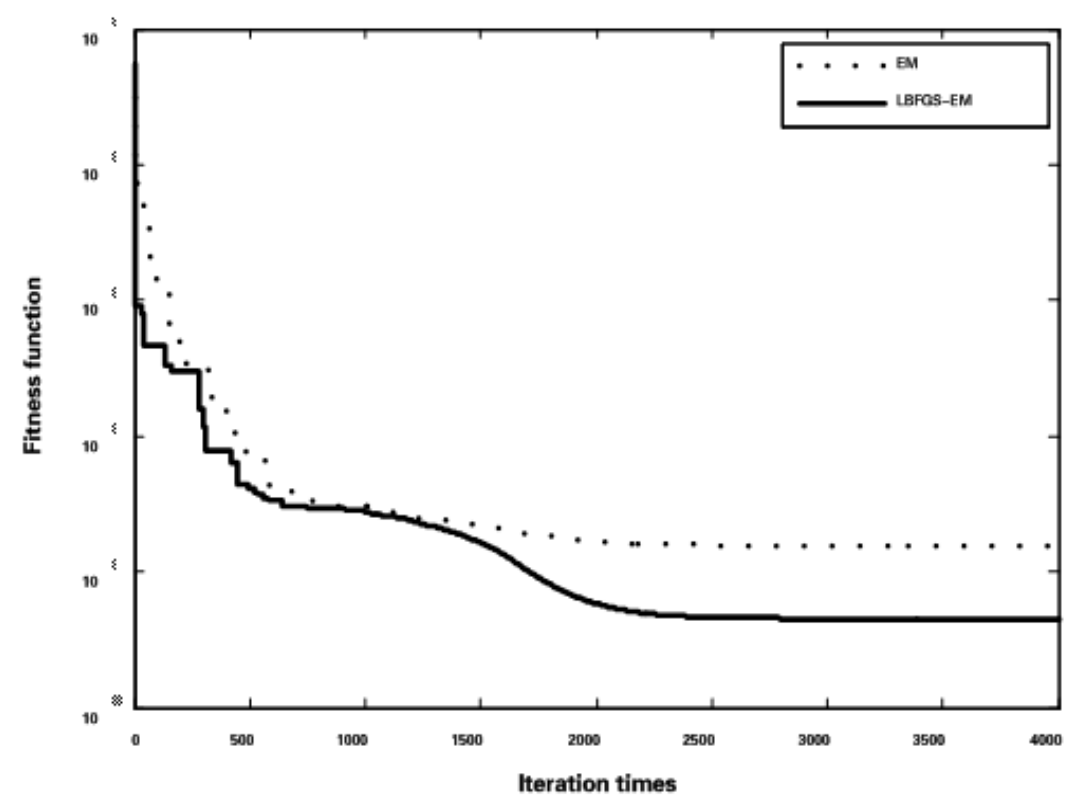

Fig. (3). ROSENBROCK test function convergence curve contrast figure.

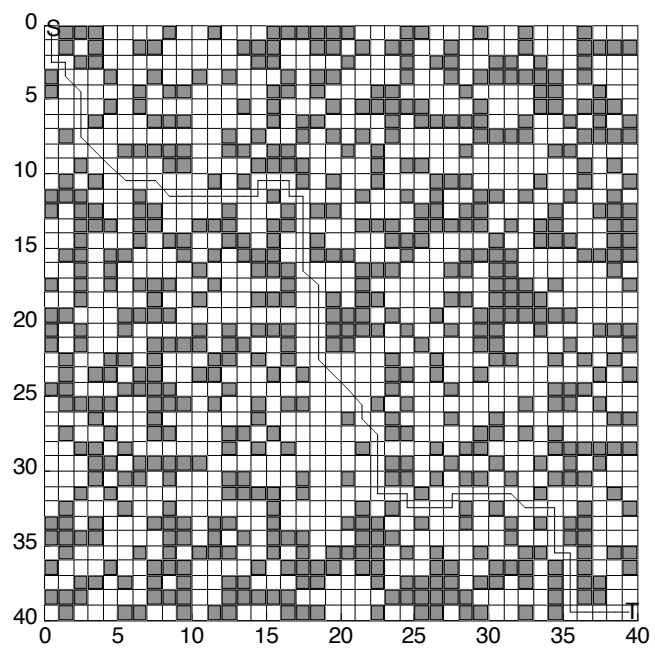

(a) Path optimization schematic diagram based on LBFGS-EM algorithm

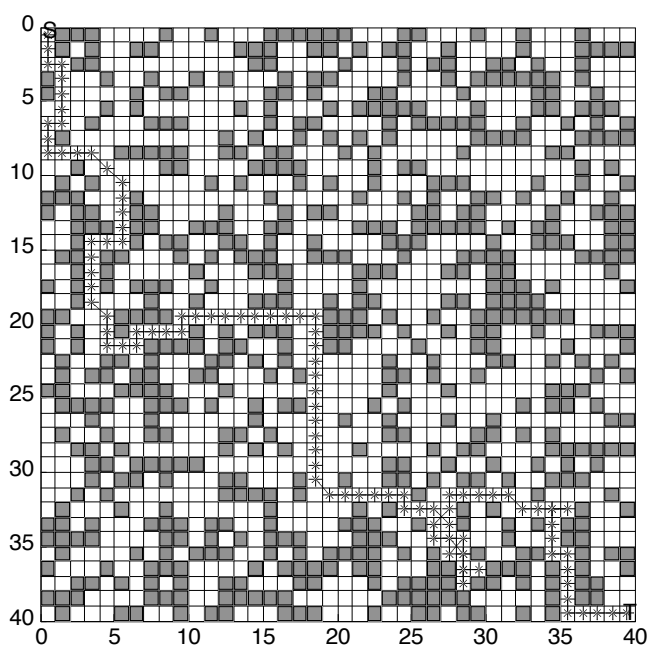

(b) Path optimization schematic diagram based on particle swarm algorithm

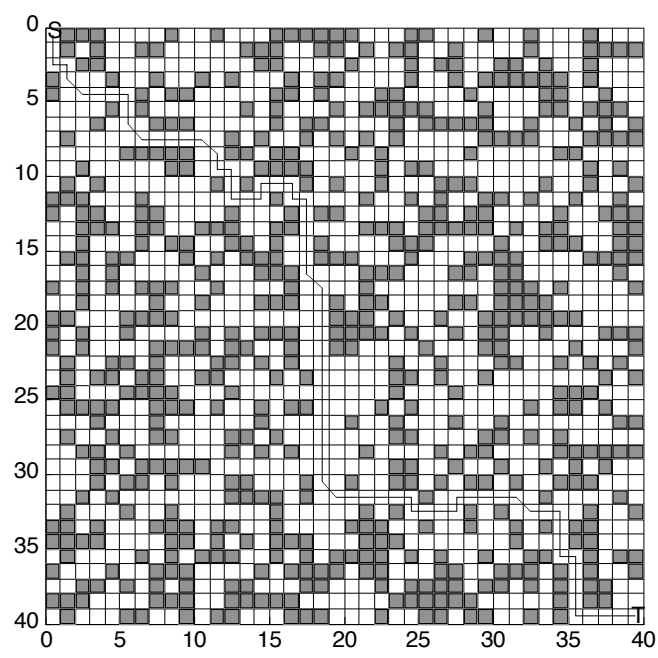

(c) Path optimization schematic diagram based on cellular ant colony algorithm

Fig. (4). Path optimization results schematic diagram. 
high quality solutions by using the test groups, so the LBFGS-EM algorithm search to the solution of higher precision after the iteration completion, more press close to the optimal solution.

In order to verify the algorithm's ability of fast convergence, this paper for the test function of the optimal individual and the known extreme value is less than a certain value, then convergence it, setting Sphere function value is 1.0e-15, GRIEWANK function value is 0.01 , ROSENBROCK function value is 0.1 , then identified as convergence. Using this algorithm, the EM algorithm [1], PSO algorithm [7], and TVAC algorithm [7-9] test take its average of 100 times for the average number of iterations, algorithm iterative number of termination for 4000 generations, get the results shown in Table $\mathbf{1}$.

In view of the test function 1 and 2, this paper proposes algorithm iterations times is the smallest, fastest convergence speed; in view of the test function 3 , until the rest of the algorithm iterations terminating also difficult to convergence, and this algorithm introduces into the confined quasi-Newton operator can effective local optimization, and uses the chaotic mapping to guide the optimization direction, so the convergence effectively, less number of iterations, fast convergence speed.

\subsection{Path Optimization Applications}

For the path optimization problem of the global map by the sensor obtain is a $0-1$ programming discrete domain optimization problem in essence. So the paper proposes the algorithm and the existing cellular ant colony algorithm [15], particle swarm optimization (PSO) to the known 40*40 maps for path planning. Get the path as shown in (Fig. 4).

Can be found from (Fig. 4a) as shown in the path is the shortest path, namely this paper's algorithm can get the optimal path, applies to optimization problem in discrete domain. To analyze its reason, the path shown in (b) the generated path is random probability event, so there will be a certain degree of "round trip" phenomenon; and the path shown in (c) although through cellular automata to avoid "round trip" phenomenon, but the ant colony algorithm optimization ability not beautiful, so get the path is not best; the algorithm is shown in this article do not need to rely on cellular automata to exclude path "round trip" phenomenon, but all path for optimization, through the algorithm itself all strong optimization ability, especially the confined quasi-Newton operator high accuracy of optimization ability, the excluded "round trip" phenomenon, to get the optimal path.

In order to verify the algorithm stability and rapidity, this paper adopts three kinds of algorithm to the above map for path planning 100 times, gets the distance and convergence time statistics as shown in Table 2, assume that each square space longer is 1 , the diagonal approximation is 1.4.

\section{CONCLUSION}

This article simply describes the basic principle of EM, and analyzes their advantages and disadvantages, in view of the local optimization ability is not strong in the late algorithm, the defect of its easy to fall into local optimum, confined quasi-Newton local optimization operator is introduced, the improved algorithm local search performance; in the iteration process, introducing the chaos operator, so that to keep the diversity of species, effectively avoid the premature phenomenon of the algorithm, have higher precision of solution. Finally the simulation results show that LBFGS-EM algorithm in continuous domain optimization, can jump out of local optimal solution, have higher precision of solution, convergence speed ideal; in the using of the path planning problem, can through the searching optimal capability of the algorithm itself and high-precision search capabilities, avoiding the "round trip" phenomenon, get the best path, and faster convergence speed, apply to the optimization problem in discrete domain

\section{CONFLICT OF INTEREST}

The author confirms that this article content has no conflict of interest.

\section{ACKNOWLEDGEMENTS}

Declared none.

\section{REFERENCES}

[1] L. X. Han, and Y. P. Wang, "Solving the unconstrained optimization problem of class electromagnetic mechanism algorithm," Electronic Journals, vol. 37, no. 3, pp. 664-668, 2009.

[2] J. G. Jiang, M. N. Liu, and Y. Q. Liu, "A kind of using double chaotic search of the class electromagnetic mechanism algorithm," Journal of Xi'an University of Electronic Science and Technology (Natural Science Edition), vo1. 41, no. 5, pp. 91-97, 2014.

[3] Z. Z. Guo, and J. K. Wu, "Power distribution network fault range positioning of electromagnetism algorithm," Journal of Chinese Electrical Device, vol. 30, no. 13, pp. 34-39, 2010.

[4] F. L. Xiong, and H. S. Yan, "Based on the strategy of leap auxiliary work mixed flow assembly line production scheduling optimization," Acta Automatica Sinica, vol. 38, no. 2, pp. 259-269, 2012.

[5] L. Liu, H. Liu, and H. Zhang, "Full waveform inversion based on modified quasi-Newton formula," Chinese Journal of Geophysics, vol. 56, no. 7, pp. 2447-2451, 2013.

[6] Y. F. Sun, C. K. Zhang, and J. G. Gao, "The chaotic parallel GA with feedback and its application in nonlinear constrained optimization," Journal of Computer, vol. 30, no. 3, pp. 424-430, 2007.

[7] X. J. Wu, Z. Z. Yang, and M. Zhao, "Even search particle swarm algorithm,” Electronic Journals, vol. 39, no. 6, pp. 1261-1266, 2011.

[8] W. B. Wang, Research and Application of Particle Swarm Optimization Algorithm CHENGDU, Southwest JIAOTONG University, 2012.

[9] A. Ratnaweera, S. K. Halgamuge, H. C. Watson, "Self-organizing hierarchical particle swarm optimizer with time-varying acceleration coefficients," IEEE Transactions on Evolutionary Computation, vol. 8, no. 3, pp. 240-255, 2004.

[10] L. Nocedal, "Updating Quasi-Newton matrices with limited storage," Mathematics of Computation, vol. 35, no. 1, pp. 773-782, 1980.

[11] J. Shu, "Authentication key agreement protocol based on extended chaotic mapping," Journal of Physics, vol. 63, no. 5, pp. 1-4, 2014.

[12] M. Liu, Z. G. Chen, and X. H. Den, "Image distort detection algorithm based on chaos and fragile watermark," Computer Application, vol. 33, no. 5, pp. 1371-1373, 2013.

[13] L. P. Liu, and X. F. Zhang, "Image encryption algorithm based on chaos and bit operations," Computer Application, vol. 33, no. 4, pp. 1070-1073, 2013. 
[14] Y. Q. Li, Y. H. Chi, and T. A. Wen, "Particle swarm optimization algorithm based on dynamic boundary," Electronic Journals, vol. 41 , no. 5, pp. 865-870, 2013.
[15] W. X. Zhang, X. L. Zhang, and Y. Li, "Based on improved particle swarm algorithm of intelligent robot path planning," Computer Application, vol. 34, no. 2, pp. 510-513, 2014.

Received: September 16, 2014

Revised: December 23, 2014

Accepted: December 31, 2014

(C) Liang Chen; Licensee Bentham Open.

This is an open access article licensed under the terms of the Creative Commons Attribution Non-Commercial License (http://creativecommons.org/licenses/by-nc/3.0/) which permits unrestricted, non-commercial use, distribution and reproduction in any medium, provided the work is properly cited. 